SUBJECT AREAS:

APPLIED PHYSICS

MATERIALS SCIENCE

Received

13 March 2014

Accepted

6 August 2014

Published

1 September 2014

Correspondence and requests for materials should be addressed to

E.B. (Edward@ariel. ac.il)

\title{
Scaling law governing the roughness of the swash edge line
}

\author{
E. Bormashenko ${ }^{1,2}$, A. Musin ${ }^{1,2} \&$ R. Grynyov ${ }^{1}$
}

'Department of Physics, Ariel University, P.O.B. 3, Ariel 40700, Israel, ${ }^{2}$ Department of Chemical Engineering and Biotechnology, Ariel University, P.O.B. 3, Ariel 40700, Israel.

The paper is devoted to the analysis of the shape of the swash edge line. Formation of the swash boundary is treated as an interfacial phenomenon. The simplest quantitative characteristic of the roughness of interface is its width $w$, defined as the root-mean-square fluctuation around the average position. For rough interfaces, the scaling with size of the system $L$ is observed in the form $w(L) \propto L^{\zeta}$. The concept of scaling supplies a simple framework for classifying interfaces. It is suggested that the fine structure of the swash boundary results from the combined action of the pinning force applied by random defects of the beach and elasticity of distorted swash boundary. The roughness of the swash front was studied at the Mediterranean Sea coast for uprush and backwash flows. Value of exponent $\zeta$ for receding swash front line was $0.64 \pm 0.02$, when in the case of advancing swash the value $0.73 \pm 0.03$ was calculated. The scaling exponent established for the receding phase of the swash is very close to the values of the exponent established for the roughness of the triple line for water droplets deposited on rough surfaces, crack propagation front in Plexiglas, and for the motion of a magnetic domain walls.

T

he physics of swash, i.e. a layer of water that washes up on the beach after an incoming wave has broken, is complicated and intriguing. It includes perplexing hydrodynamic and sediment transport events ${ }^{1-4}$. The coastal strip is a highly dynamic zone and may include features with the dimensions ranging from microns to kilometers. Beach cusps are often arising in the swash zone presenting obstacles to the propagation of a wave front and promoting its breakdown ${ }^{1-4}$. In our paper, we address the roughness of the moving swash boundary at which a beach, water and air meet. The roughness of the swash edge is characterized by a scaling law that relates the statistical width $w$ of the boundary (i.e. the root-mean-square fluctuation of the edge line around its average position) to its length $L$ via $w(L) \propto L^{\zeta} \mathrm{law}^{5-10}$. We treat the behavior of this boundary as an interfacial phenomenon, without going into details of the formation of edge waves and beach cusps, covered broadly in literature ${ }^{1-4}$. This "crude" approach turns out to be productive and reveals the resemblance of the swash line to a broad diversity of effects arising from the random pinning of moving boundaries ${ }^{5-10}$.

Swash consists of two phases: uprush (onshore flow) and backwash (offshore flow). We concentrate mainly on backwash (offshore flow), when water recedes along the beach face. The receding water front is pinned by randomly distributed obstacles, produced by the beach and constituting the roughness of the swash edge line. It is plausible to suggest that the fine structure of the swash edge line results from the combined action of the pinning force applied by defects of the beach and the elasticity of the distorted swash boundary. The problem is common for a number of physical systems comprising interfaces moving in a disordered medium; for example, self-affine crack propagation $^{5-6}$, wet front propagation and movement of interfaces between immiscible liquids in porous media ${ }^{7-9}$, height distribution of growing surfaces ${ }^{10,11}$, and growth of magnetic domain walls in disordered media ${ }^{12}$. However, the most investigated situation of interfaces moving over a disordered system of obstacles is the displacement of the triple (three-phase) line at which water, vapor and a rough (or chemically heterogeneous) surface meet ${ }^{13-17}$.

The shape of a triple line disturbed by the presence of random heterogeneities on the solid surface may be derived by a solution of the Laplace equation with the boundary conditions of the Young equation. With some simplifications, this is given by Shanahan ${ }^{18}$ and de Gennes ${ }^{19}$. The simplest quantitative characteristic of an interface is its width $w$, defined as the root-mean-square fluctuation around an average position. For rough interfaces, scaling with size of a system $L$ is observed, which is expressed in the form

$$
w(L) \propto L^{\varsigma},
$$

meaning that the average width $w$ grows as the size $L$ is increased. The concept of scaling introduced in the field of interfaces by Family and Vicsek ${ }^{20,21}$ gave a simple framework for classifying interfaces in various processes. Scaling 


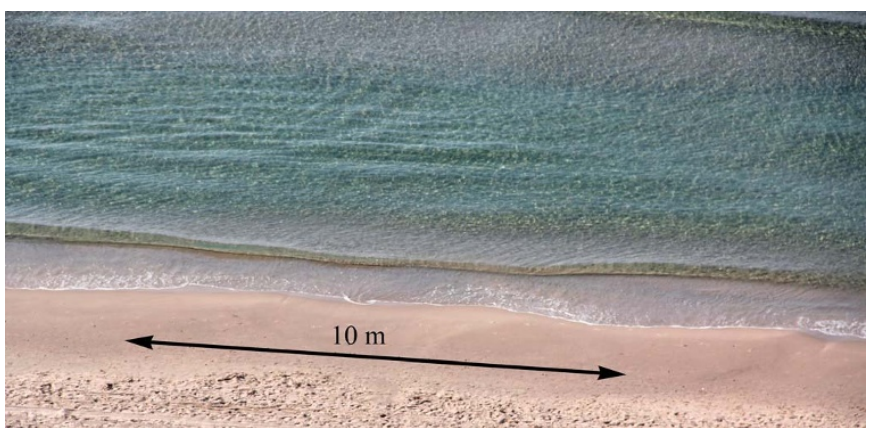

Figure $1 \mid$ Propagation of water front and receding swash edge (the picture is taken by R. Grynyov at the Mediterranean Sea coast).

laws describing the shape of the triple line were studied in many works ${ }^{13-16}$. A constant value of the roughness exponent $\zeta$ in a given physical system was proposed to express some universal law (independent on the topography of a relief but influenced by the ratio between the statistical width $w$ and the length scale of obstacles), and gave rise to different models. Exponent values 1/2 and 1/3 (for fluctuations smaller and larger than the length scale of defects, respectively) were calculated by Robbins and Joanny ${ }^{22}$ and Joanny and de Gennes $^{23}$, according to the model of long-range elastic forces. It was demonstrated in Refs. 22-23, that deformation of a wave vector $k$ characterizing the contact line will distort the surface of the liquid over a distance $k^{-1}$ (for the "normal" elasticity the distortion will scale as $k^{-2}$ ). Assuming that the surface deforms instantaneously in response to the contact-line distortions, the elastic energy cost for the deformation can be calculated from the surface tension energy stored in the distorted area, and it is thus proportional to $|k|$.

The values of the roughness exponents derived by Robbins and Joanny ${ }^{22}$ were supposed to be universal for describing contact lines on surfaces with disordered defects. These values indeed were observed by Rolley et al. ${ }^{14}$ in their experiments with superfluid helium- 4 on a cesium substrate with random disorder. Values of about 0.5 were measured for a water meniscus on a glass plate covered with random spots of chromium ${ }^{15}$. On the other hand, values of the roughness exponent measured in other experiments differed from the given theoretical predictions. Values in the region 0.82 0.87 were measured for water, and $0.77-0.86$ for hexadecane on a chemically degraded fluorinated polymer surface ${ }^{13}$. Experiments performed with helium-4 deposited on a strongly disordered cesium substrate $^{24}$ supplied the roughness exponent $\zeta=0.56 \pm 0.03$. The value of $\zeta=0.63$ appeared in a number of different models, based on directed percolation ${ }^{25}$. This value was obtained for a wet front propagation in porous media ${ }^{8}$, and for the crack propagation fronts in plastics $^{26,27}$.
In our recent work ${ }^{28}$, we measured the roughness exponent of the triple line of a water droplet deposited on a porous polymer (polycarbonate) substrate in a situation of the Cassie wetting, and we calculated the values $0.60 \pm 0.05$ for the receding triple line and $0.63 \pm 0.02$ for the advancing triple line. It was plausible to suppose that the exponent value $\zeta=0.6-0.63$ is universal of for the processes when a mobile water interface is stopped by randomly distributed pinning sites, forming so-called capillary fringes ${ }^{4,19}$. We suggested, that the backwash phase of swash fulfils these demands, regardless of the nature of the beach. Thus, the value of the scaling exponent is expected to be the same as or close to the value established for triple lines. We checked this hypothesis experimentally. It is noteworthy, that the universality of the exponent is kept until the roughness is smaller than the correlation length of the defects (pinning sites), thus, we restricted our research by this domain of roughnesses ${ }^{14,22}$.

\section{Results and Discussion}

Several hundreds of pictures of a water front and swash edge were taken at the Mediterranean Sea coast on a fair and calm day (wind speed $0.95 \mathrm{~m} / \mathrm{s}$, height of waves $0.21 \mathrm{~m}$ ); see Fig. 1. Two states, uprush (onshore flow) and backwash (offshore flow), presenting advancing and receding swash edges, may be distinguished, and all images were classified according to these two cases.

Positions of swash edges on the images were digitized according to the method described earlier ${ }^{28}$ and characterized by coordinates $x$ along an edge and $y$ in the perpendicular direction (Fig. 2).

Characteristic lengths featuring the shape of the swash edge were evaluated with its Fourier expansion, defined by the coordinates $y$ and $x$ as:

$$
y(x)=\sum_{m} a_{m} e^{i k_{m} x}, \quad k_{m}=2 \pi / \lambda_{m},
$$

where $a_{m}$ are expansion coefficients corresponding to wavelengths $\lambda_{m}$. Figure 3 presents modules of the expansion coefficients $a_{m}$ depending on the wavelengths $\lambda_{m}$. According to Fig. 3, the dominant contribution to the roughness of the swash edge is given by the wavelengths in the range of 6-8 m.

It is noteworthy that the dominant wavelengths established in our experiments coincide with "typical beach cusp" lengths $s^{2,29}$.

Roughness exponents of swash edges were calculated according to the method described earlier ${ }^{15,16,28}$. A section of a swash edge with a total length $D=17 \mathrm{~m}$ was expressed by $n$ points, with coordinates $y_{i}$ (width) and $x_{i}$ (length) differing by the value $\Delta x=6.3 \mathrm{~cm}$. It should be emphasized that studied sections of a swash edge were larger than the dominant wavelengths of the Fourier expansion, calculated above. Segments with the length $L(L<D / 2)$ including $n(L)$ points were chosen, and for each segment its rms width $\sigma(L)$ (roughness) was calculated according to:
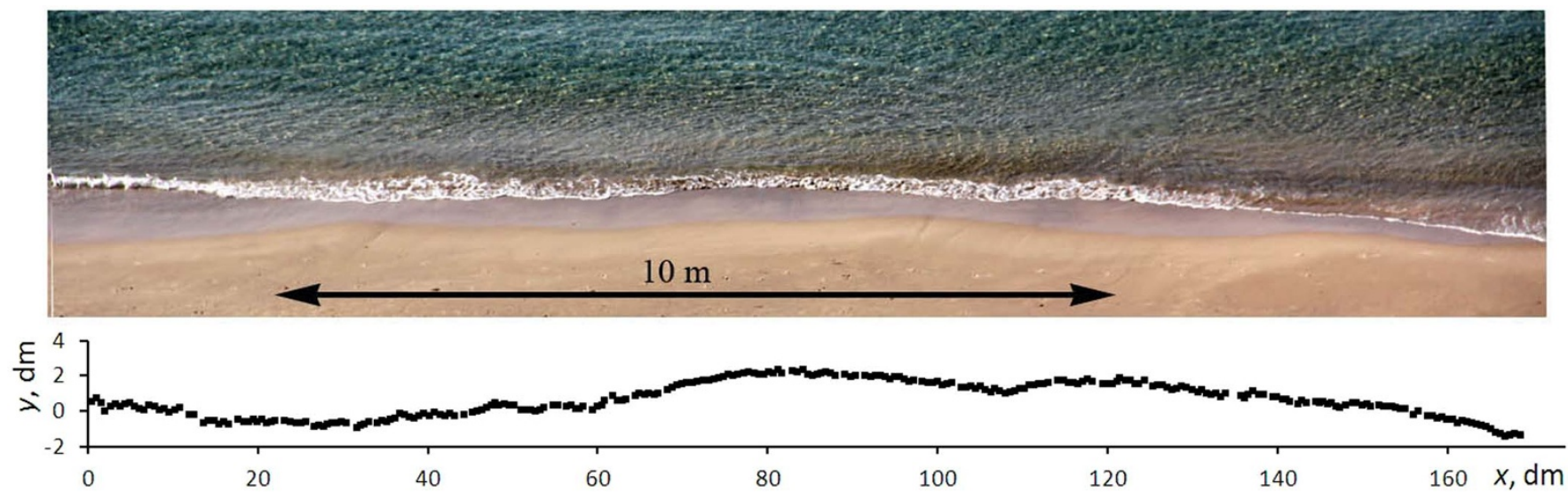

Figure $2 \mid$ Advancing swash edge and its digitized presentation $y(x)$ (the picture is taken by R. Grynyov at the Mediterranean Sea coast). 


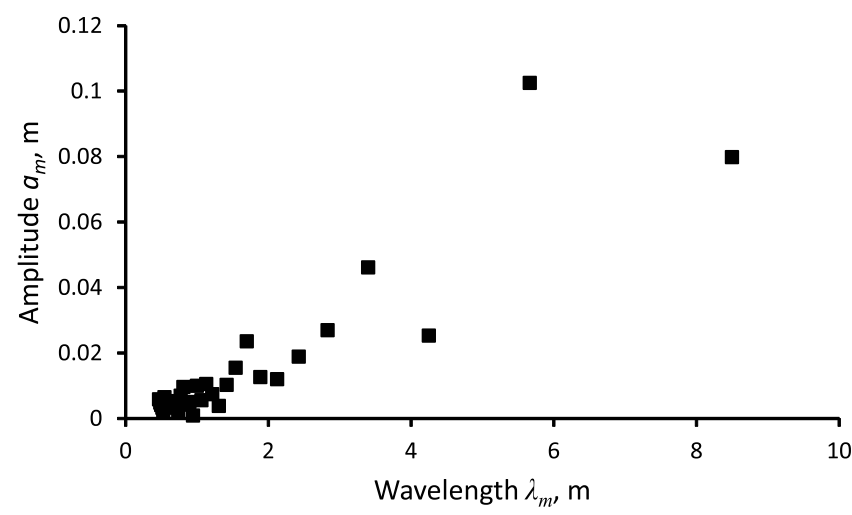

Figure 3 Typical Fourier spectra of a receding swash edge.

$$
\begin{gathered}
\sigma^{2}(L)=\frac{1}{L} \sum_{i=1}^{n(L)}\left[y_{i}-\bar{y}(L)\right]^{2} \Delta x, \\
\bar{y}(L)=\frac{1}{L} \sum_{i=1}^{n(L)} y_{i} \Delta x .
\end{gathered}
$$

After that, the average roughness $w(L)$ of an ensemble of segments with the same $L$, centered in successive points of swash front $x_{0 j}$, was calculated according to the equation:

$$
w(L)=\left(\frac{1}{D-L} \sum_{j} \sigma^{2}\left(L, x_{0 j}\right) \Delta x_{0 j}\right)^{0.5},
$$

$\Delta x_{0 j}$ being the distance between the centers. Finally, the roughness of the swash edge was characterized by its average rms width $w$ dependent on the length $L$. Dependences $w(L)$ presented in Fig. 4 are finely approximated with the scaling law $w(L) \propto L^{\zeta}$. The value of exponent $\zeta$ for the receding swash edge was $0.64 \pm 0.02$, whereas in the case of advancing swash the value $0.73 \pm 0.03$ was calculated.

It should be stressed that the derived values of roughness exponents were obtained for the values of roughness $(w<0.12 \mathrm{~m})$ much smaller than the typical beach cusp lengths ${ }^{2,29}$. It is well-known that the switch of the roughness exponent occurs when the roughness approaches to the correlation length of the defects ${ }^{14,22}$. However, due to experimental restrictions we did not enter into this area, and did not observe the switch of exponent $\zeta$.

The values of the scaling exponent $\zeta$ established for the receding phase of the swash are very close to the value of the exponent $\zeta=0.60$ \pm 0.05 , established for the roughness of the triple line for water

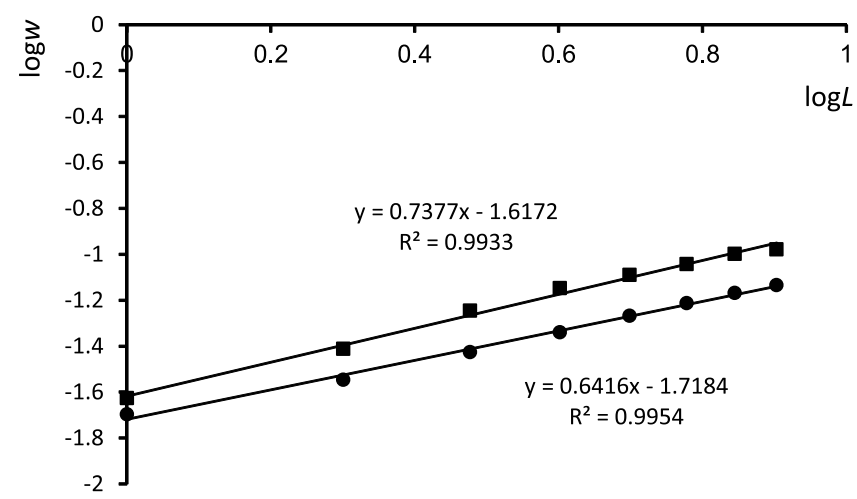

Figure $4 \mid$ Typical graphs of the average roughness $w$ versus length $L$ for advancing (squares) and receding (circles) swash edges. droplets deposited on rough surfaces ${ }^{28}$. The same exponent arises for a crack propagation front in a heterogeneous Plexiglas ${ }^{27}$, and for the motion of a magnetic domain walls ${ }^{12}$.

This coincidence deserves discussion. It may be suggested that the swash front, the triple line and a crack propagation front may be described as randomly disturbed elastic strings ${ }^{27,30-31}$. Rosso and Krauth $^{30}$ demonstrated that the anharmonic elastic potential of a randomly disturbed string, containing the second and fourth powers of the string displacement, leads to almost the same value of $\zeta$ equal to 0.63 . Moreover, the same exponent appears in models based on directed percolation ${ }^{25}$. In these models, the mobile interface is stopped by a directed percolation cluster of pinning sites ${ }^{25}$. At the same time, the scaling law appropriate for advancing swash is characterized by the higher value of $\zeta=0.73 \pm 0.03$. It is plausible to relate this discrepancy to the dependence of advancing swash on the hydrodynamics of running waves. Indeed, the advancing swash edge is formed not only by pinning events, but also by the dynamics of bounding waves.

In the future investigations the swash boundaries demonstrating roughness approaching the correlation length of defects should be studied. Thus, the switch of the roughness exponent is expected ${ }^{14,22}$. Swash edges formed by beaches of various morphologies also call for study $^{32,33}$.

1. Huntley, D. A. \& Bowen, A. J. Field observation of edge waves. Nature 243, 160-162 (1973).

2. Guza, R. T. \& Inman, D. Edge waves and beach cusps. J. Geophys. Res. 80, 2997-3012 (1975).

3. Werner, B. T. \& Flink, T. M. Beach cusps as self-organized patterns. Science 260, 968-971 (1993).

4. Horn, D. P. Measurements and modelling of beach groundwater flow in the swash-zone: a review. Cont. Shelf Res. 26, 622-652 (2006).

5. Schmittbuhl, J. \& Måløy, K. J. Direct observation of a self-affine crack propagation. Phys. Rev. Lett. 78, 3889-3891 (1997).

6. Rice, J. R. Fist-order variation in elastic fields due to variation in location of a planar crack front. J. Appl. Mech. 52, 571 (1985).

7. Rubio, M. A., Edwards, C. A., Dougherty, A. \& Gollub, J. P. Self-affine fractal interfaces from immiscible displacement in porous media. Phys. Rev. Lett. 63, 1685 (1990).

8. Horvath, V. K., Family, F. \& Vicsek, T. Dynamic scaling of the interface in twophase fluid flows. J. Phys. A 24, L25 (1991).

9. Buldyrev, S. V. \& Barabasi, A.-L. Anomalous interface roughening in porous media: experiment and model. Phys Rev. A 45, R8313-R8316 (1992).

10. Plischke, M., Racz, Z. \& Zia, R. K. P. Width distribution of curvature-driven interfaces: a study of universality. Phys. Rev. E 50, 3589-93 (1994).

11. Tong, W. M., Williams, R. S., Yanase, A., Segawa, Y. \& Anderson, M. S. Atomic force microscope study of growth kinetics: scaling in the heteroepitaxy of $\mathrm{CuCl}$ on $\mathrm{CaF}_{2}$ (111). Phys. Rev. Lett. 72, 3374 (1994).

12. Lemerle, S. et al. Domain wall creep in an Ising ultrathin magnetic film. Phys. Rev. Lett. 80, 849 (1998).

13. Decker, E. L. \& Garoff, S. Contact line structure and dynamics on surfaces with contact angle hysteresis. Langmuir 13, 6321-6332 (1997).

14. Rolley, E., Guthmann, C., Gombrowicz, R. \& Repain, V. Roughness of the contact line on a disordered substrate. Phys. Rev. Lett. 80, 2865-2868 (1998).

15. Moulinet, S., Rosso, Al., Krauth, W. \& Rolley, E. Width distribution of contact lines on a disordered substrate. Phys. Rev. E 69, 035103R (2004).

16. Moulinet, S., Guthmann, C. \& Rolley, E. Roughness and dynamics of a contact line of a viscous fluid on a disordered substrate. Eur. Phys. J. E 8, 437-443 (2002).

17. Bormashenko, Ed. Wetting of Real Surfaces (De Gruyter, Berlin, 2013).

18. Shanahan, M. E. R. Disturbed triple line behavior and its use in surface analysis. Adv. Colloid Interface Sci. 39, 35-59 (1992).

19. de Gennes, P. G., Brochard-Wyart, F. \& Quéré, D. [Hysteresis and elasticity of triple line]. [69-85] Capillarity and Wetting Phenomena (Springer, Berlin, 2003).

20. Vicsek, T. \& Family, F. Dynamic scaling for aggregation of clusters. Phys. Rev. Lett. 52, 1670-1672 (1984).

21. Family, F. \& Vicsek, T. Scaling of the active zone in the Eden process on percolation networks and the ballistic deposition model. J. Phys. A 18, L75 (1985).

22. Robbins, M. O. \& Joanny, J. F. Contact angle hysteresis on random surfaces. Europhys. Lett. 3, 729 (1987).

23. Joanny, J. F. \& de Gennes, P. G. A model for contact angle hysteresis. J. Chem. Phys. 81, 552-562 (1984).

24. Prevost, A., Rolley, E. \& Guthmann, C. Dynamics of a helium-4 meniscus on a strongly disordered cesium substrate. Phys. Rev. B. 65, 064517 (2002).

25. Kardar, M. Nonequilibrium dynamics of interfaces and lines. Phys. Rep. 301, 85-12 (1998). 
26. Santucci, S., Grob, M., Toussaint, R., Schmittbuhl, J., Hansen, A. \& Måløy, K. J. Fracture roughness scaling: a case study on planar cracks. Europhys. Lett. 92, 44001 (2010).

27. Delaplace, A., Schmittbuhl, J. \& Måløy, K. J. High resolution description of a crack front in a heterogeneous Plexiglas. Phys. Rev. E 60, 1337-1343 (1999).

28. Bormashenko, E., Musin, A., Whyman, G., Barkay, Z. \& Zinigrad, M. Revisiting the fine structure of the triple line. Langmuir 29, 14163-14167 (2013).

29. Dolan, R. \& Ferm, J. C. Crescentic landforms along the mid-Atlantic coast. Science 159, 627-629 (1968).

30. Rosso, A. \& Krauth, W. Origin of the roughness exponent in elastic strings at the depinning threshold. Phys. Rev. Lett. 87, 187002-1-4 (2001).

31. Rosso, A. \& Krauth, W. Roughness at the depinning threshold for a long-range elastic string. Phys. Rev. E 65, 025101R (2002).

32. Wright, L. D. \& Short, A. D. Morphodynamic variability of surfzones and beaches: a synthesis. Marine Geology 56, 93-118 (1984).

33. Masselink, G., Hegge, B. J. \& Pattiaratchi, Ch. B. Beach cusp morphodynamics. Earth Surface Processes and Landforms 22, 1139-1155 (1997).

\section{Author contributions}

G.R. took pictures, M.A. made pictures processing and calculated the roughness exponents, B.E. analyzed data and draw conclusions. All authors discussed the results and reviewed the manuscript.

\section{Additional information}

Competing financial interests: The authors declare no competing financial interests.

How to cite this article: Bormashenko, E., Musin, A. \& Grynyov, R. Scaling law governing the roughness of the swash edge line. Sci. Rep. 4, 6243; DOI:10.1038/srep06243 (2014)

This work is licensed under a Creative Commons Attribution-NonCommercialNoDerivs 4.0 International License. The images or other third party material in this article are included in the article's Creative Commons license, unless indicated otherwise in the credit line; if the material is not included under the Creative Commons license, users will need to obtain permission from the license holder in order to reproduce the material. To view a copy of this license, visit http:// creativecommons.org/licenses/by-nc-nd/4.0/ 